\title{
INVESTIGAR, ESCRIBIR Y ENSEÑAR HISTORIA \\ EN LA ERA DE INTERNET. PRESENTACIÓN
}

\author{
FRANCISCO FERNÁNDEZ IZQUIERDO \\ Instituto de Historia, CSIC.
}

RESUMEN: Las nuevas tecnologías de la información (NTIC) y comunicaciones se han desarrollado en grado sumo en la sociedad postindustrial, que por ello se califica como sociedad de la información. La informática, las bases de datos, y finalmente Internet, suponen un nuevo medio en el que un oficio tradicional, como es el de los historiadores, tiene que buscar su acomodo. A partir de la última década del siglo XX, y especialmente desde 1995 se han publicado artículos y monografías relatando experiencias apoyadas en NTIC, tanto en la investigación, como en la docencia en niveles básicos y universitarios, se ofrecen guías sobre las producciones multimedia y las páginas web de contenido histórico surgidas en los paises más desarrollados. No obstante, pese al apoyo oficial y la inversión en nuevos medios, los historiadores continúan sirviéndose preferentemente de sus fuentes tradicionales (libros, artículos, documentación de archivo), pero no dudan en aplicar las ventajas que ofrecen los sistemas modernos de acceso a la información, cuando favorecen el desarrollo del discurso historiográfico y el debate.

Palabras Clave: Historia. Nuevas tecnologías de la información. Internet. Historiografía. Enseñanza universitaria. Enseñanza media. Bases de datos. Ediciones multimedia.

\begin{abstract}
The new information and communication technologies (ICT) have increased at a high level in postindustrial society, which is described as an information society for that reason. Computer science, the data bases, and finally the Internet, suppose new means in which a traditional profession, as is the one of the bistorians, must look for its arrangement. Since the last decade of XXth century, and specially from 1995, articles and monographs have been published relating experiences supported in ICT, dealing with research, and teaching in basic and university levels, and offering guides on the multimedia productions and web pages of historical content published in developed countries. However, in spite of the official support and the investment in new tools, historians continue using their traditional sources (books, articles, documents from archives), but they do not fail to apply the advantages offerd by modern systems of access to information, when they help in the development of the historiographic discourse and the debate.
\end{abstract}

KEY WORDS: History. Information technologies. Internet. Historiography. University teaching. Basic teaching. Databases. Multimedia editions. 
Aunque la economía mundo de la que hablaba I. Wallerstein en la década de 1970 tuvo su origen en la expansión ultramarina europea del siglo XVI, la globalización no se ha percibido como un hecho cotidiano hasta que los medios de masas transformaron las comunicaciones humanas más allá de las fronteras físicas y políticas en el siglo XX, culminando al final de esta centuria en la etapa calificada como sociedad postindustrial o sociedad de la información. La aparición de Internet como red de comunicación global ha superado y democratizado todo lo conocido hasta entonces. Mientras que a principios de los años 90 del siglo XX las nuevas tecnologías de la información y de las comunicaciones (conocidas por sus siglas españolas NTIC o en inglés IT) estaban aún limitadas a los ámbitos de investigación de vanguardia radicados en los laboratorios académicos y militares donde se gestaron estos avances, una década y media más tarde, en 2006, Internet ha supuesto una verdadera revolución en las relaciones y comunicaciones humanas, afecta a los mercados financieros y económicos que reaccionan en tiempo real a escala planetaria, influye en las decisiones políticas de los Estados, y también se ha introducido en la vida cotidiana con la popularización de las páginas web, el correo electrónico, los chats, blogs... Aunque su apertura data de principios de la década de 1990, la verdadera difusión popular de Internet acaba de cumplir apenas diez años, los que se inician a mediados de esa década ${ }^{1}$. Sobre la base de redes informáticas previas, limitadas por los protocolos de comunicación o su tecnología, en los años 80 el primer salto hacia una nueva red abierta partió desde algunos departamentos y laboratorios universitarios a la totalidad de los campus, pasando por las bibliotecas y centros de documentación. Pero la diseminación llegó también a las grandes empresas, los organismos públicos, extendiéndose luego a la enseñanza media y primaria, para alcanzar finalmente al público en general gracias a la rebaja de costes en las conexiones por cable, y al aumento de la capacidad de los accesos a Internet mediante ADSL en cualquier parte a la que llega el cable telefónico, e incluso más allá, gracias las conexiones inalámbricas tipo WiFi.

Los humanistas, y los historiadores en particular, manejamos información como fuente principal de nuestro discurso. De acuerdo con el pensamiento tecnológicamente correcto el valor de los nuevos medios de comunicación es incuestionable, pues acceder más rápidamente a más datos debería mejorar los resultados de la actividad humana, y por extensión la del historiador, hasta niveles mucho más elevados que los alcanzados sirviéndonos de los métodos artesanales empleados en recopilar y analizar informaciones. ¿Es esto realmente cierto? La respuesta debería ser afirmativa, pero con matizaciones, muchas más que pocas. En el día de hoy si se interrumpe el acceso a la red informática el trabajo intelectual queda seriamente afectado, puesto que en la red es donde se en-

1 Estos años coinciden con las fechas de publicación de la trilogía de CASTELLS, Manuel: La era de la información. Madrid: Alianza, 1996-1998. Más recientemente, NORMAN, Jeremy M. (ed.): From Gutenberg to the Internet: a sourcebook on the history of information technology, Novato, Calif.: Historyofscience.com, 2005. 
cuentran los catálogos en línea de las bibliotecas académicas y nacionales, los inventarios de cada vez más archivos, y los portales especializados en Internet desde donde los correos electrónicos nos asaetean con noticias y novedades, con consultas y respuestas en listas de discusión, o con las últimas ocurrencias de un blog. Sin embargo, la transmisión de conocimiento sólo puede alcanzarse tras la lectura pausada de materiales selectos, la reflexión sobre su contenido, y la síntesis destilada a partir de la cosecha recogida en un bosque de información cada vez más espeso, intrincado e inabarcable.

En una primera etapa, durante la segunda mitad de los años 80 y en los primeros 90, cuando la información se movía aún lentamente en las redes telemáticas, el CD-ROM alcanzó el primer puesto en la difusión de grandes cantidades de información. La mejora en la digitalización y el aumento de capacidad, disminución de coste y facilidad de producción era un acicate para volcar en estos nuevos medios los repertorios y textos que habían sido objeto de recopilación y de estudio durante siglos ${ }^{2}$. Los DVD multiplicaron la capacidad de los antiguos discos, pero especialmente el aumento del ancho de banda en las redes ha convertido en innecesaria la edición de ejemplares individuales, cuando la información puede consultarse en tiempo real. Nos encontramos en un punto en el que las facilidades para la difusión son contrarias a la defensa de los derechos de propiedad intelectual (y su explotación económica) en que se sustentan los editores científicos, lo que impide el acceso gratuito a muchas publicaciones. Frente a las suscripciones onerosas y el peaje obligado para acceder a bases de datos con materiales científicos, está ganando crédito el movimiento denominado Open Access, la publicación libre de derechos de cada vez más información. Esto no es ajeno a las compañías más destacadas en Internet. Cuando Google puso en marcha su sección académica Google Scholar, en noviembre de 2004, apuntaba un crecimiento notable en sus contenidos ${ }^{3}$, al tiempo que en diciembre de ese año acuerdos entre esta compañía y diversas bibliotecas universitarias, entre ellas la de la Universidad de Harvard, pretendían ofrecer el texto completo de millones de libros, siguiendo tendencias aplicadas por los principales libreros de la red, como Amazon, que también está digitalizando los libros actuales para facilitar su comercialización. Frente a los esfuerzos generalizados en volcar información en Internet cuanto más amplia mejor, como ayuda para disponer de lo que hasta ahora se encuentra en repertorios impresos o inéditos, y una creencia en que ello facilitará el desarrollo del discurso historiográfico, la propia información que se puede obtener en la red nos puede facilitar en qué medida está afectando este fenómeno a la tarea de los historiadores

2 Hay información sobre múltiples proyectos en este sentido, véase como ejemplo: BRENNER, M.L.: «The Lincoln Legal Papers and the new age of documentary editing», Computers and the Humanities 30 (5), 1996, pp. 365-372.

3 JACSO, N.: "Google Scholar: the pros and the cons», On line information Review, 29 (2), 2005, pp. 208-214 
académicos ${ }^{4}$. Sirviéndonos de las principales bases de datos que contienen referencias a artículos científicos pertinentes con el asunto analizado, hemos realizado varias consultas que se describen a continuación y cuyas cifras manifiestan el peso relativo de los resultados obtenidos.

Bases de datos de Thompson - ISI, conocidas como Web of Knowledge $e^{5}$, estos resultados:

bistorians and computing, 18 documentos (sólo 9 relativos al uso de ordenadores por historiadores, el resto son de historia del cálculo o de la computación)

bistorians and comput*, 68 documentos

bistorian* and Internet, 20 resultados

history and Internet, 647 resultados (la mayoría referidos a la propia historia de Internet, no al uso de Internet para difundir temas históricos)

historians and web* 62 resultados (algunos ya localizados arriba, y la mayoría referidos al uso de "web" como red — no informática—, o a los nombres Max Weber o Webb)

La base de datos Historical Abstracts de $\mathrm{ABC} \mathrm{Clio}^{6}$, ofreció los siguientes resultados:

history and Internet: 132

database: 437

teaching history and computers: 127

Se han consultado otras bases de datos y portales de acceso a información bibliográfica, especialmente libros, en busca de una visión general que completase la ofrecida por los artículos?

4 El manejo eficiente de las bases de datos referenciales (bibliográficas) o en texto completo que se ofrecen hoy en día en la red precisa de herramientas adecuadas para enfrentarse a la diversidad de formatos, y al volumen de información que hay que tratar. Algunas recomendaciones al respecto pueden obtenerse en Collins, Susan L.: «In the aggregate: a quantitative study of the impact of interdisciplinary full text databases on historical research», Journal of the Association for History and Computing 6(1), 2003.

5 http://portal.isiknowledge.com consultando de forma conjunta en SCI-expanded, SSCI, A\&HCI. Todas las consultas de este trabajo se han verificado en el segundo trimestre de 2006.

6 http://www.abc-clio.com. Aunque las bases de datos del ISI son las preferentes en ciencias, la historia en general y la especialización en países distintos al ámbito cultural anglo-americano está mucho mejor reflejada en otras bases de datos como Historical Abstracts. Esta situación podría cambiar cuando sea una realidad el European Reference Index on the Humanities (ERIH), en el que trabaja un equipo de la Fundación Europea de la Ciencia desde 2003, con apoyo económico de la Unión Europea.

7 La base de datos ISOC, mantenida por el CINDOC, dependiente del CSIC, en http://bddoc.csic.es:8085. Francis, base de datos sobre humanidades y ciencias sociales, producida por el INIST en Francia. http://www.inist.fr/PRODUITS/francis.php También se han consultado el catálogo colectivo de las bibliotecas universitarias españolas, Rebiun, http://bibliotecnica.upc.es/ 
De manera equivalente a lo que puede observarse en otras muchas actividades que implican comunicación en el mundo actual, Internet está ganando cuota de audiencia entre los historiadores, tanto entre los profesionales situados en niveles universitarios y de investigación ${ }^{8}$ como entre los docentes de los niveles medios y primarios, así como entre el público ávido de que le cuenten historia. Ya en la primera mitad de los años 90, los historiadores profesionales se hicieron eco tempranamente de las posibilidades del nuevo medio ${ }^{9}$, que a finales de la década ya alcanzaba una madurez suficiente como para reflexionar sobre su potencialidad para difundir resultados. Parecía no haber límite para las investigaciones necesitadas de canales baratos y capaces de comunicar grandes cantidades de datos, como son los recopilados y elaborados con la minuciosidad y erudición propia de los especialistas, cuya forma tradicional de trabajo se veía favorecida por la nueva tecnología, en abierta contradicción con algunas de las ideas manejadas en el debate del fin de la historia ${ }^{10}$. La capacidad de la informática para almacenar, clasificar y realizar búsquedas en grandes corpora de información textual, gráfica o multimedia, superaba todo lo conocido hasta la implantación y aumento de la potencia en ordenadores y medios de almacenamiento digital. La utilidad de las bases de datos en estudios históricos no admite discusión, y hay abundantes testimonios en la literatura científica. Con precedentes incluso anteriores, ya desde los años 70 del siglo $\mathrm{XX}$ el contacto entre las ciencias históricas, la informática y las bases de datos ha quedado definido en la historiografía ${ }^{11}$, aunque gracias a la generalización de las herra-

Rebiun/nova/principal/index.asp y el acceso virtual a bibliotecas nacionales de todo el mundo, y catálogos colectivos que ofrece la Universidad de Karlsruhe, desde Alemania, http://www.ubka.unikarlsruhe.de/kvk_span.html. Existen muchas otras bases de datos, a las que hace referencia Cruz Rubio en su artículo en este monográfico.

8 JENKS, Stuart; MARRA, Stephanie (eds.): Internet-Handbuch Geschichte. Köln: Böhlau, 2001, X+294 p. MeYer, Thomas; BurckHARDT, Daniel; BeIER, Gerhard: «Digitale Medien in der historischen Forschung», Information Wissenschaft und Praxis, 54 (4)2003, pp. 235-237, correspondiente a la referencia del congreso "Geschichte and neue Medien" (Historia y Nuevos medios) Berlin 9-11 abril 2003.

9 MitTaG, Jürgen; SAHLE, Patrick: «Geschichte und Computer im Internet: Informationsgewinnung zwischen Chaos und Ordnung», Historical Social Research, Köln, 21, 2 (1996), pp. 126-132.

10 Jochum, Uwe: «Das Ende der Geschichte im Internet» [Recurso electrónico]: (Vortrag Basel, 30.03.2001, für die Tagung "Raumlose Orte - geschichtslose Zeit"), [S.l.]: Universität Konstanz / Zentrale Einrichtungen, Bibliothek, 2004. Publicado en Geschichte und Informatik 21 (2001), p. 1121 [http://www.bsz-bw.de/cgi-bin/xvms.cgi?SWB11051689]. Uhde, Karsten: «20012010: Gegenwart und Zukunft des Internet als Gemeinsame Arbeitsplattform von Archivaren und Historikern: «Arbeitstagung Internet: "Raumlose Orte - geschichtslose Zeit"), Arbido, 16 (6), 2001, pp. 9-14.

11 Una recopilación de 173 títulos desde 1953, la mayoría posteriores a 1995, se encuentra en SCHLEIGH, Amy: «At the intersection of history and technology: a bibliography for historians and information professionals», Journal of the Association for History and Computing, 5(3), 2002. Otro informe, relativo al período 1989-1998, en GERSMANN, Gudrun: «Neue Medien und Geschichtswissenschaft: ein Zwischenbericht», Geschichte in Wissenschaft und Unterricht, 50(4), 1999, pp. 239-249. 
mientas ofimáticas desde la década de 1980, todos los historiadores las utilizan en sus investigaciones sin apenas hacer mención a ello. Sólo cuando algún proyecto lo requiere por su dimensión, complejidad o particularidad, los autores inciden en sus publicaciones y hacen mención de este instrumento informáti$\mathrm{CO}^{12}$. La tecnología más eficiente es la que apenas se nota: simplemente se usa.

El nuevo medio, Internet, apenas cuestionado, cuenta con un apoyo militante de gobiernos e industrias, se le considera el canal de moda, pero no por ello está falto de limitaciones. Entre ellas, la volatilidad de los materiales publicados en Internet preocupa a los historiadores y archiveros, pues muchas páginas desaparecen sin dejar rastro al retirarse los servidores desde los que se publican, mientras que su conservación sería útil para analizar el tiempo presente $^{13}$. Igualmente el contexto de producción en los materiales difundidos en el ciberespacio no está tan formalizado como en las publicaciones impresas o audiovisuales, se diluye en la red ${ }^{14}$. Por otra parte, la digitalización masiva y el acceso en la red no mejoran necesariamente los resultados de los proyectos de investigación, pues contamos con escasa información sobre qué aplicaciones hacen los historiadores de los archivos digitales, en la línea de los estudios de

12 En Historical Abstracts, la consulta "database*” ofreció 437 resultados. La relación entre bases de datos y publicación en la red ofrece algunos ejemplos tan tempranos como Bernhist, base de datos con indicadores demográficos, económicos y sociales del cantón suizo de Berna entre 1700 y 1990, que se abrió a consulta en Internet desde finales de 1994. IMFELD, Klaus; PFISTER, Christian; HABERLI, Peter: «Bernhist: Eine raum-zeitliche Datenbank für den schweizer Kanton Bern im Internet», Historical Social Research, 20(3), 1995 20(3), pp. 102-133. Otro ejemplo es el uso compartido de una base de datos relativa a autores europeos de historia y política entre los siglos XIV y XVII en GENET, Jean-Philippe: «Les bases de données historiques à distance: Une experience», Le Médiéviste et l'ordinateur. 41, 2002, pp. 83-86. Diversas ediciones electrónicas se describen en CHIOcChETTI, Filippo: «Fonti storiche, fonti digitalizzate, metafonti. Qualche nota su alcune iniziative recenti», Storia della Storiografia, (44), 2003, pp. 104-120. Las referencias a la oferta de bibliografías, archivos y otros repertorios documentales son numerosas. Por otra parte, la consulta combinando "history and database*" en Web of Knowdledge, sólo ofreció 167 resultados, en los cuales la mayor parte se refieren al estudio de la historia natural (clima, terremotos, evolución de especies biológicas), a estudios de tipo demográfico, genético o clínico, o sobre los efectos humanos sobre el medio ambiente, temas que son novedosos entre los historiadores, aunque cada vez aparecen más como objetivos para equipos de investigación interdiscipinares. Como ejemplo, uno relativo a la historia del clima, en DIETRICHFELBER, U.: «Using Java and XML in interdisciplinary research - A new data-gathering tool for historians working with EuroClimHist», Historical Methods 37 (4), otoño 2004, pp. 174-185. Una visión general epistemológica sobre la historia, apoyada en las posibilidades de la codificación informática con XML y las técnicas de inteligencia artificial puede verse en BERNARDO ARES, J.M. de; Calvo Cuenca, A.: Historia e informática. Metodología interdisciplinar de la investigación bistórica. Córdoba: Servicio de Publicaciones de la Universidad - Cajasur, 2005, 344 pp.

13 También la tecnología puede suplir estas pérdidas, pues motores de búsqueda como Google son capaces de almacenar en su memoria caché páginas incluso dessparecidas. El debate sobre la volatilidad de la información electrónica se encuentra también entre las prioridades de los archiveros, como responsables de la custodia de lo que será mañana documentación histórica.

14 LOR, P.J.; BRITZ J.; WATERMEYER H.: «Everything, for ever? The preservation of South African websites for future research and scholarship», Journal of Information Science, 31 (1), 2006, pp. 39-48. 
usuarios que acostumbran a realizar los responsables de las actuales bibliotecas y centros de documentación sobre los servicios que prestan, con cuyos resultados se pretende orientar mejor el destino de los recursos disponibles ${ }^{15}$.

La representación del pasado, durante mucho tiempo elaborada en su discurso por los cronistas e historiadores, a la que se sumaba la pintura histórica y cualquier otra iconografía artística con tintes historicistas, en la presente era digital está escapando cada vez más de las manos de los profesores de historia. La creatividad que antes se expresaba en forma de obras literarias, se potencia con la libertad que proporcionan hoy los recursos multimedia e Internet, recreando espacios que se hacen realidad gracias a la proliferación de juegos informáticos, y a las representaciones virtuales que también se han introducido en la cinematografía. El pasado se reconstruye con gran cantidad de detalles y profusión de medios, pero en términos y con objetivos alejados de los que buscan los investigadores académicos ${ }^{16}$. No obstante, los mismos instrumentos favorecen que la representación y reconstrucción virtual de ambientes históricos tenga cada vez más aceptación cuando corre a cargo de especialistas en patrimonio, arqueólogos, historiadores del arte, arquitectos y otros profesionales que se sirven de las técnicas de modelado en 3D, fotogrametría, infografía, animación y procedimientos imposibles de realizar con el nivel de sofisticación y realismo al que estamos acostumbrados, si no fuera gracias a la actual potencia de los ordenadores y de los programas informáticos ${ }^{17}$. Los profesionales de los archivos y los museos, los responsables de la gestión del patrimonio histórico y cultural, han asumido la tarea de ofrecer en Internet no sólo sus tradicionales catálogos e instrumentos de descripción, sino presentar los contenidos que gestionan mediante exposiciones virtuales, en busca de una mayor repercusión social ${ }^{18}$.

15 EVERITT, S.: «Opportunistic collaboration - Unlocking the archives of the Birmingham Institute of Art and Design», Program-Electronic Library and Information Systems, 39 (4), 2005, p. 312-323. Un proyecto desarrollado en la Universidad de Goteborg (Suecia) se describe en BENNER, M.: «The digital archive of the Swedish East India Company, 1731-1813: a joint project of a university library and a history department», Online Information Review, (5), 2003, pp. 328-332.

16 Rietbergen, P.: «Representations of the past in word, image and game. A complex cultural continuum beyond the scholarly text», Tijdschrift Voor Geschiedenis 117 (2), 2004, pp. 187-206.

17 Paquet, E., VikTOR, H.L.: «On the application of 3D technologies to the framework of cultural heritage» Annales des Télécommunications-Annals of Telecommunications 60 (11-12), nov-dic 2005, pp. 1379-1393.

18 REID, G.: «The digitisation of heritage material: arguing for an interpretative approach based on the experience of the Powys Digital History Project» Program-Electronic Library and Information Systems 34 (2), abr. 2000, pp. 143-158. NeNTWIG, L.: «LeMO: A virtual exhibition of 20th century German history», Computer Networks-The International Journal Of Computer And Telecommunications Networking 31 (21), nov. 1999, pp. 2237-2244. MARTY, P.F.: «On-line exhibit design: The sociotechnological impact of building a museum over the World Wide Web», Journal Of The American Society for Information Science, 51 (1), 2000, pp. 24-32. WEIDNER, Marcus: «Westfälische Geschichte im Internet - Projektbericht zum Internet-Portal 'Westfälische Geschichte': ein Kooperationsprojekt des 
¿Cómo está afectando esta situación a la práctica diaria del historiador, en sus facetas de docente, investigador y escritor? Hemos de remitirnos, en primer lugar, a diversos trabajos entusiastas de los recursos que las nuevas tecnologías de la información ofrecen al investigador, al profesor y al estudiante, junto a debates abiertos respecto a los cambios que suponen en la forma de crear y transmitir los conocimientos históricos ${ }^{19}$. Mientras algunos autores se han ocupado de redactar guías o manuales breves sobre los recursos en Internet interesantes para los historiadores ${ }^{20}$, otros han pretendido ofrecer un panorama global de esta nueva forma de difusión en monografías extensas, algunas con CDROM incluidos, y con tal éxito editorial que ha permitido la aparición de ediciones actualizadas en años sucesivos ${ }^{21}$. A partir de la segunda mitad de la dé-

Westfälischen Instituts für Regionalgeschichte und der Stiftung Westfalen-Initiative», Westfälische Forschungen 5, 2003, pp. 447-475. [http://www.lwl.org/westfaelische-geschichte/portal/Internet/] Un proyecto australiano intentando recrear los viajes de Cook se describe en TURNBULL, Paul: «Engaging with history complexity in the virtual environment: the South Seas project.», Archives and Manuscripts [Australia] 30(1), 2002, pp. 66-81. Otro en el que se asocia la investigación y publicación de fuentes con nuevas tecnologías en soporte informático es el de LóPEZ-Ocón, Leoncio; Ponsati, Agnès (dirs.): La Comisión Científica del Pacífico de la expedición al ciberespacio: 1862-1866 - 1998-2003 [Recurso electrónico] Madrid: Consejo Superior de Investigaciones Científicas, Ministerio de Educación y Ciencia, Una y Media, 2004. 1 disco (DVD), y su versión en Internet http://www.pacifico.csic.es/uym3/default.htm.

19 WeLSCH, E.K.: «The wired historian, Internet prospect and problems», Centennial Review, 38 (3), otoño 1994, pp. 479-502. FerRAND, N.; Delon, Michel, (dirs.): «Nouveaux instruments de la recherche; Bilans et perspectives de la recherche», Dix huitième siècle: Paris. (30), 1998, pp. 293306. Dacos, Marin: «Un historien au pays du minitel: le web et l'histoire en France a la fin de l'année 2000", Journal of the Association for History and Computing 2000, 3(3). HABER, Peter: Geschichte und Internet: "raumlose Orte - geschichtslose Zeit" = Histoire et Internet, Zürich: Chronos, 2002, 177 p. MINUTI, Rolando: Internet et le métier d'historien: réflexions sur les incertitudes d'une mutation. Paris: Presses Universitaires de France, 2002, 146 p.

20 EDER, Franz X.; TANTNER, Anton. «Internet fur Historiker / Innen». Österreichische Zeitschrift fur Geschichtswissenschaften [Austria] 1995 6(2), pp. 325-330, (3), pp. 453-456, (4), pp. 604-608. Internet et histoire: dossier. [Bruxelles: CEGES], [1999] 28 p. FRIEDHEIM, William: A Web of connections: a guide to history on the Internet. Boston, Mass.; London: McGraw-Hill College, 1999. VII+54 pp. SOARES, John: Internet guide for history, Belmont, Calif.; London: Wadsworth $2^{\circ}$ ed., 2000, 63 pp. CHANDLER, Fiona: The Usborne internet-linked first encyclopedia of history. London: Usborne, 2003, $64 \mathrm{p}$.

21 Horvath, Peter: «Geschichte Online: Neue Möglichkeiten für die historische Fachinformation», Historical Social Research (Supplement 8): 1997, pp. 1-283. DITFURTH, C.V.: Internet für Historiker, Frankfurt/Main; New York: Campus-Verlag, 1997, con nuevas ediciones actualizadas en 1998 y 1999. OHRMUND, Andreas; TIEDEMANN, Paul: Internet für Historiker: eine praxisorientierte Einführung. Darmstadt Primus-Verlag, 1999, con reed. actualizada en 2000. TRINKLE, Dennis A.: The World History highway: a guide to Internet resources. Armonk, N.Y.: M.E. Sharpe, 1997, XII+249 pp. Ediciones posteriores actualizadas de esta obra a cargo de TrinkLE, Dennis A, y MERRIMAN, Scott A., (eds.), se han publicado en 2002 y la última en mayo de 2006. Para la historia europa, de TRINKLE, Dennis A, y MERRIMAN, Scott A., (eds.): The European history highway a guide to internet resources, New York; London: M.E. Sharpe, 2002, VIII+279 p + 1 CD-ROM. GROSCH, Waldemar: Geschichte im Internet. Tipps, Tricks und Adressen. Schwalbach/Ts.: Wochenschau-Verl., 2002, 167 pp. Sorprendentemente, estos libros apenas están presentes en la bibliotecas españolas, incluidas las universitarias. 
cada de 1990 son frecuentes las publicaciones de noticias sobre información encontrada en Internet, seleccionada por las áreas académicas de las ciencias históricas ${ }^{22}$, temáticas (v.g. historia económica, historia de la Iglesia y las religiones, historia militar, estudios de género, biografía, historia del deporte...) $)^{23}$, o geográficas ${ }^{24}$. Desde entonces, cada vez es más habitual encontrarse anuncios

22 Por ejemplo, para Historia Antigua pueden mencionarse numerosos trabajos: GiLLILAND, Anna Marie: An evaluative review of Internet resources for students of classics, ancient bistory, and classical archaeology. Tesis (M.A.) University of Sheffield, Department of Information Studies, 1998. KaUfmann, Dieter; TIEDEmanN, Paul: Internet für Althistoriker und Altphilologen: eine praxisorientierte Einfübrung. Darmstadt: Primus-Verl., 1999. FONTES BlanCO-LoIZELIER, Fernando L.: «Guía española de recursos en Internet sobre Prehistoria, Arqueología, Historia Antigua y gestión del patrimonio», Complutum (8), 1997, pp. 374-385. GOMEZ-PANTOJA, Joaquín; LÓPEZ Trujillo, Miguel A.: «En las redes de Clío. Historia Antigua e Internet: un caso practico», Memoria y Civilización. Anuario de Historia, (1) 1998, pp. 79-96. Recursos de información en Internet sobre arqueología, prehistoria e historia antigua: CINDOC, 8 y 9 de mayo de 2000. [Madrid]: CINDOC, 2000, 28 p. Sehlmeyer, M.: «CD-ROMs and Internet sources for the study of the history of late antiquity and the Middle Ages», Historische Zeitschrift. 274 (2), 2002, pp. 367-386. DONATI GiACOMINI, Paola: Innovazione e tradizione: le risorse telematiche e informatiche nello studio della storia antica. Bologna: Il Mulino, 2002, 137 p. AlvonI, Giovanna: Scienze dell'antichità per via informatica: banche dati, Internet e risorse elettroniche nello studio dell'antichità classica Bologna: Clueb, 2002. XVIII, 195 p. MÜlLER, Matthias: Alte Geschichte online: Probleme und Perspektiven althistorischen Wissenstransfers im Internet. Sankt Katharinen: Scripta Mercaturae Verl., 2003, X+127 pp. En lo relativo a Historia Moderna, y otras áreas en España, dimos cuenta en FERNÁNDEZ IZQUIERDO, F.: «La Historia Moderna y Nuevas Tecnologías de la Información y las Comunicaciones», Cuadernos de Historia Moderna, (24), 2000, pp. 207-238.

23 LEPORE, Amedeo: «Le fonti elettroniche per la storia economica», Cromobs: Cyber Review of Modern Historiography, 8, 2003. GómeZ DíAz, Donato: «La Historia Económica en Internet», Historia Actual On-line, 1(2) 2003. HuCK, S.: «Military history on the internet», Militargeschictliche Mitteilungen 59 (1), 2000, pp. 206-209. DíEZ De Velasco Abellán, Francisco P.: «Recursos en Internet para la investigación y la docencia en historia de las religiones», Ilu, revista de Ciencias de las Religiones, 1996, 39-52. STRICKLAND, Michael: «Some selected internet resources for novice researchers of Christian History», Journal of Religious E Theological Information, 20013 (3-4), pp. 151-160. VAN SLUIS, J.: «Kerkgeschiedenis op Internet, mogelijkheden en beperkingen» Nederlandsch theologisch tijdschrift. 54 (3), 2000, pp. 238-248. SLATTA, R.W., HAYWOOD E.K.: «Enhancing Latin American history teaching and research with computers», Social Science Computer Review, 23 (2), Sum 2005, pp. 152-166. BARREIRO, N.: «Internet resources for feminist literary historians», Nouvelles Questions Feministes 22 (2), 2003, pp. 117-121. SENTILLES, R.M.: "Catching it all on the web: Crafting cohesive American women's history in the age of the Internet» Journal of Womens History 16 (2), 2003, pp. 175-177. Freeman, S.K.; GuY, D.J.; HewitT, N., et al.: «Perspectives on teaching women's history - Views from the classroom, the library, and the Internet» Journal of Womens History 16 (2), 2004, pp. 143-176. FRESQUET FEBRER, José Luis: «Las TIC (Tecnologías de la Información y la Comunicación) y los estudios biográficos», Asclepio. Revista de Historia de la Medicina y de la Ciencia 57(1), 2005, pp. 167-187. Cox, Richard William; Salter, Michael A.: «The IT revolution and the practice of sport history: an overview and reflection on Internet research and teaching resources», Journal of Sport History 25(2), 1998, pp. 283-302.

24 Como muestras veamos algunas referencias fundamentalmente de países occidentales. EVERETT, James E.: «Annual review of information technology developments for economic and 
de nuevas páginas aparecidas en la red, o comentarios sobre portales especializados en Historia ${ }^{25}$, noticias que por su frecuencia suelen comentarse de forma

social historians, 1996», Economic History Review [Great Britain] 50(3), 1997, pp. 543-555. BROMUND, Ted R.: «A guide to planning and conducting research in Contemporary British history on the Internet», Contemporary British History 16(4): 2002, pp. 109-122. NASH, S.D.; YOSHIMURA, M.; VINCENTI, W.: "American history resources on the Internet», College and research libraries news. 57 (2) 1996; 82-84, 96 [4 p.]. NASH, S.D.; DíAZ, Karen R., (eds).: «Reference sources on the Internet in United States history; Reference sources on the Internet: off the shelf and onto the Web», The Reference librarian. (57)1997, pp. 167-178. CONNER, Susan P.: «The web we weave: Search engines, databases and other internet resources for historians of France», Proceedings of the Annual Meeting of the Western Society for French History 1999, 26, pp. 63-75. RUBIO LINIERS, M. C.; Ruiz Franco, M. del Rosario; Andrés Verdú, R. de; Bustelo Toretlla, J.: «Internet y la historia de España», Revista General de Información y Documentación, 8 (2), 1998, pp. 149-163. MONTAÑA CONCHIÑA, Juan Luis de la «Aportaciones de las N.T.I.C. al mundo de la enseñanza y la investigación: Internet y sus aplicaciones en la Historia», Campo Abierto, (16), 1999, pp. 37-54. FERNÁNDEZ GARCÍA, Jesús: «Recursos para la investigación de la historia actual en Internet», Simposio de Historia Actual. IV. 2002. Logroño: 2004, vol. 1, pp. 337-352. DÖRR, Marianne: «Fachinformation Geschichte im Internet: Angebote der Bayerischen Staatsbibliothek», Jabrbuch der historischen Forschung in der Bundesrepublik Deutschland, München, 2001 (2002), pp. 63-68. KeLLNER, Stephan: «Bayerische Geschichte im Internet: neue Möglichkeiten für die Heimatforschung». Forum Heimatforschung, München 1996, 9 (2004), p. 51-56. AвBATTISTA, Guido; ChiocCHetTI, Filippo: "An outline survey of Italian historiography in the world wide web». History and Computing, 12(3), 2000, pp. 287-305. CRISCIONE, Antonino et al. (eds.): La storia a (l) tempo di internet: indagine sui siti italiani di storia contemporanea, 2001-2003. Bologna: Patron, 2004, 387 p. Bosco, Giuseppe: «Didattica della storia: Torino nella Seconda Guerra Mondiale», Contemporanea: Rivista di Storia dell'800 e del '900, 6(4), 2003, pp. 751-757. UGAI, Masashi: «Nihon no rekishigaku kenkyu ni okeru inta-netto riyo no genjo» [The current state of Internet use for historical research in Japan], Rekishi Hyoron [Japan] (6): 1998, pp. 24-34. Brown, Philip C.: «The Internet and historical studies in Japan: a personal view, Perspectives», American Historical Association Newsletter 36(9), 1998, pp. 45-50. TANKARD, K.: «History, the Internet and South Africa», Suid-Afrikaanse Historiese Joernaal-South African Historical Journal (38), 1998, pp. 20-33. KLEIN, Herbert and LUNA, Francisco Vidal: «Sources for the study of Brazilian Economic and Social History on the Internet», Hispanic American Historical Review 84(4), 2004, pp. 701-715. LU, Zhenguang: "Yintewang shang shixue xinxi ziyuan cutan» [A preliminary inquiry into historiographical data on the Internet]. Shixue Yuekan (Journal of Historical Science) [China] (4) 1999, pp. 2-7. «Istorik, istochnik i Internet» [Historians, sources, and the Internet], Novaia i Noveishaia Istoriia [Rusia] (2), 2001, pp. 66-93.

25 FISHER, D.: «Navigational aids for the history of science, technology and the environment www.nahste.ac.uk (Review of Internet site)», History Today, (1), ene. 2003, p 61. DeverGE, M.: «It's Byzantine! (Byzantium internet sites, ancient history)», Histoire (242), abr. 2000, p. 93. Id.: «Internet resources (Maritime history)» Histoire, nro. esp. 8, jun 2000, pp. 107. «On the Web (Internet sites of interest to readers of 'American History'», American History, 35 (3), ag. 2000, p. 6. SPONSLER, C.: «All the World's a Stage: WWW links for theatre history and early music by Abigail Young (Internet publication)», Theatre Journal, 51 (4) dic. 1999, pp. 478-479. FITZGERALD, I.: «Multimedia history (A listing of resources available on the Internet, video, and CD-ROMs)», History Today, 47 (12), 1997, pp. 52-55. RUNDLE, D.: «Internet history (A survey of the latest multimedia innovations, are they useful to historians)», History Today 48 (11), 1998, pp. 14-15. THUillier, J.: «The computer and art history + CD-ROM, Internet research», Revue de l'Art, (117), 1997, pp. 5-8. DiamOND E.; ABTES, S.: "The Ancient-History of the Internet », American Heritage 46 (6), oct. 1995, p. 34 . HorowiTZ, M.: «Finding History on the net + Internet » American Heritage 46 (6), oct. 1995, p. 44-45. 
similar a las recensiones de libros en muchas revistas, tanto en las profesionales como en las de divulgación. El nacimiento de revistas electrónicas serias y, más en particular, de portales especializados en los que los enlaces y la información suministrada se somete a selección, ofrece ciertas garantías para orientarse en un panorama caótico como es el de Internet ${ }^{26}$. La vinculación entre la enseñanza universitaria y las nuevas tecnologías se describe en algunos artículos que reflexionan sobre cómo Internet y las páginas web, o el uso del correo electrónico se han implantado de manera generalizada en los departamentos de Historia ${ }^{27}$, de la misma forma que en todas las organizaciones académicas. No obstante, frente a iniciativas puntuales muy vanguardistas que exploran en las posibilidades de las nuevas tecnologías ${ }^{28}$, los cursos a distancia y otras experiencias lectivas con la red como base aunque se comenten, por el momento alcanzan un impacto mucho menor que los métodos docentes tradicionales ${ }^{29}$. Conocer y desenvolverse en estas técnicas es una de las destrezas que hay que

26 KiTCHENS, J.D.: «Clio's new clothes: Electronic journals for history and some considerations for reference service», Internet reference services quarterly, 4(2), 1999, pp. 69-77. Refiriéndose a la revista electrónica Tiempos Modernos http://www.tiemposmodernos.org/ sobre la utilidad de este tipo de sitios postula Téllez AlARCiA, Diego: «La recopilación de recursos electrónicos en línea de alta calidad científica: una propuesta metodológica para historia moderna» Revista general de información y documentación, 13 (1) 2003, pp. 77-95.

27 «French history e-mail internet information list», French Historical Studies 22 (4), otoño 1999, pp 654. Talja, S., Savolainen, R., Maula, H.: «Field differences in the use and perceived usefulness of scholarly mailing lists», Information Research-An International Electronic Journal, 10 (1): oct. 2004 art. No. 200. La aplicación del correo electrónico e Internet en la docencia de Historia Antigua se comenta en GUNNEWIG, Beatrix: «Uber den Einsatz der neuen Medien in der alten Geschichte», Geschichte in Wissenschaft und Unterricht 52(1), 2001, pp. 40-43. BALTA I MONER, Jordi: «Internet y educacion: posibilidades para la enseñanza de la historia», Educación y Biblioteca. Revista Mensual de Documentación y Recursos Didácticos (70), 1996, pp. 41-45.

28 O'Sullivan, Una: «Examining historians' use of the Internet», History and Computing, 9(13), 1997 pp. 36-42, ofrecía un análisis a la altura de 1997 del uso de Internet por los miembros del proyecto Electronic Seminars in History (ESH) en el marco del Institute of Historical Research's (IHR) de Gran Bretaña.

29 Gran Bretaña ha sido un país pionero en estas experiencias. WYNNE, Ann: «History instruction and the Internet: a literature review». Journal of the Association for History and Computing, 2 (1)1999. Para un país avanzado como Canadá, véase CORBEIL, P.: «History and the computer in Canadian institutions - An overview», Social Science Computer Review, 23 (2), Sum 2005, pp. 181-189. CRAVER, Kathleen W.: Using Internet primary sources to teach critical thinking skills in history, Greenwood professional guides in school librarianship, Westport, Conn.: Greenwood Press, 1999, XX+257 p. Un experimento docente sobre el plan urbanístico de Chicago de 1909 se comenta en DeNNIS, B., SMITH, C., Sмith, J.: «Using technology, making history: A collaborative experiment in interdisciplinary teaching and scholarship», Rethiking History, 8 (2), 2004, pp. 303-317. Sobre enseñanza de Historia a distancia en España, Collado Medina, José; Gómez BArroso, José Luis: «Aplicaciones de Internet en la Enseñanza a Distancia: la Página Web del Departamento de Economía Aplicada e Historia Económica de la UNED», RIED. Revista Iberoamericana de Educación a Distancia, 2 (1)1999, pp. 139148. En Cuba: NOA Silverio, Luisa A.: «Experiencias con Multimedios en Programas a Distancia», RIED. Revista Iberoamericana de Educación a Distancia, 3(1), 2000, pp. 131-151. 
cultivar en los futuros historiadores, pues el acceso que permite Internet o las ediciones en soporte informático a las fuentes, su comparación y el desarrollo de un espíritu crítico son cualidades que se deben estimular gracias a los nuevos medios ${ }^{30}$. A pesar del esfuerzo económico invertido, sin embargo, los historiadores apenas citan los recursos electrónicos en sus publicaciones, porque aún son muchísimos más los libros y artículos que se publican en papel. En una investigación que estamos llevando a cabo sobre las citas en las principales revistas especializadas en Historia Moderna que se publican en España y en una selección de actas de congresos publicadas en los años 2000 y 2001, entre más de 68.000 referencias bibliográficas extraídas, tan sólo 7 correspondían a páginas web, y sólo una hacía referencia a una base de datos ${ }^{31}$. Este hecho no se debe a un cierto atraso en la implantación de nuevas tecnologías entre los historiadores españoles, pues se ha comprobado la misma situación en una investigación donde se analizaban publicaciones históricas aparecidas en países occidentales entre 1995 y $2000^{32}$. Para el historiador, ni todo está en la red, ni lo que se encuentra allí es suficientemente fiable, frente a las fuentes tradicionales y a su necesaria crítica ${ }^{33}$.

La aplicación de estas producciones en la enseñanza secundaria también está sirviendo para que los alumnos se planteen problemas históricos y desarrollen su capacidad personal en investigación y reflexión ${ }^{34}$, y están disponibles algunas tesis doctorales que valoran la eficacia de los nuevos medios en la ense-

30 Spaeth, D.A; Cameron, S.; Chambers, Ellie, (eds.): "Computers and resource-based history teaching: A UK perspective; Computers in Humanities Teaching and Research: Dispatches from the Disciplines», Computers and the Humanities, 34 (3), 2000, pp. 325-343. Dallo, Alain: "Quelle informatique enseigner aux historiens? L'exemple de l'Universite de Paris-I», Le Médiéviste et l'ordinateur, 42, 2002, pp. 89-92. Yi, Hua: «Using the Internet to teach access to Asian history resources», Reference services review. 30(1), 2002, pp. 62-66.

31 Por el momento, la información de este proyecto se ofrece en http://161.111.141.93/emc

32 Graham, Suzanne R.: «Historians and electronic resources: patterns and use», History and Computing, Journal of the Association for History and Computing, 5 (2) 2002.

33 Así lo afirmaba MiLLER, Heather Lee: «Getting to the source: the world wide web of resources for women's history». Journal of Women's History 11(3): 1999 pp. 176-187. Enseñar los criterios para valorar la fiabilidad de la información que está en la red es tan importante como la instrucción en las herramientas que permiten moverse en Internet para realizar búsquedas y aplicarlas como apoyo a la clase de historia. NOONAN, Kathleen M.: «Untangling the web: the use of the world wide web as a pedagogical tool in history courses». History Teacher 31(2),1998, pp. 205-219.

34 No faltan algunas guías para los profesores. GRANDBASTIEN, J. F.: «Les nouvelles technologies de l'information et de la communication en histoire et geographie: l'usage d'Internet: Les enseignants et les technologies d'information et de communication dans l'enseignement (TICE)», Education et formations. (56) 2000, pp. 143-147, 181 [6 p.]. En la enseñanza secundaria en Escocia el apoyo tanto de iniciativas británicas como europeas en mejorar la implantación de las nuevas tecnologías en los centros, no ha conseguido aún cambios significativos en los métodos docentes en lo que a Historia se refiere. Hillis, P.; MUNRO, B.: «ICT in history education Scotland and Europe» Social Science Computer Review, 23 (2), Sum 2005, pp 190-205. Véase el amplio contenido del portal Spartacus Educational, http://www.spartacus.schoolnet.co.uk. 
ñanza ${ }^{35}$. En esta corriente de inversión en nuevas tecnologías se ofrecen guías de Internet para los docentes ${ }^{36}$, al mismo tiempo que las referencias a información en la red ya están presentes en los propios libros de texto escolares, que vienen dotados de CD-ROMs de apoyo ${ }^{37}$. En diversos autores españoles se observa la misma admiración que han expresado los profesores de otros países respecto a las posibilidades de estos medios, junto a la necesidad de adaptar su uso a los programas formativos, especialmente en la enseñanza elemental ${ }^{38}$. Los

35 ACUN, Ismail: Changing history and geography teaching with ICT: the impact of the Internet (Tesis doctoral). Leicester: University of Leicester, mayo 2003. FAWCETT, Keith: A study into the influence of the Internet and collaborative learning on the traditional school history project Tesis (M.Ed.) University of Sheffield, Division of Education, 2000. MALASZCZYK, Mark: "Global History via the Web: A Model of Instruction for Teaching Global History in the New York State Secondary School Core Curriculum.» DAI 62(11), 2002: 3892-A. DA3034925, St. John's U. 139 pp.

36 LANCASTER, John: On-line history: using the Internet in history. Dunstable: Belair, 2000, 48 pp. Avrand-Margot, Sylvia; Magret-Chelot, Anne: Les 500 sites Internet: histoire-géographie, éducation civique. Paris: Belin, 2001 y reed en 2002, 2003 y 2004, 250 p. ACHARD, Gilles; IsARD, Christian; MOLINIER, Bernard [et al.]: J'enseigne avec l'internet en histoire-géographie, ClermontFerrand: CRDP d'Auvergne, 2001, 115 p. RYGIEL, P; GuICHARD, Eric, (ed.); BEGuIN, Daniel; Benoit, Serge; Boisivon, Francois; Caro, Paul; Desbois, Henri; Di Cosmo, Roberto; Ducourtieu, Christine; Jeanneret, Yves; Mathias, Paul; Quatravaux, Dominique; RYgiel, Philippe: «L'offre faite aux historiens: Essai de description des services Web offerts aux professionnels de l'histoire contemporaine; Internet et les chercheurs». En Rapport Menrt, 95-K-6438, Ministère de l'Éducation Nationale, de la Recherche et de la Technologie, Paris, France, 1998, pp. 56-61. Verband der Geschichtslehrer Deutschlands, Landesverband Rheinland-Pfalz, und der Fachzeitschrift Praxis Geschichte - Landesverband Rheinland-Pfalz, con la participación de FIEBER, Klaus (ed.): Wegweiser durch das Internet für den Geschichtsunterricht, [recurso electrónico en CD-ROM], Braunschweig: Westermann, 2001. LOBELLO, Maria: Studiare con internet storia. Milano: Hoepli, 2000, 159 p.

37 La consulta en la base de datos del ISBN, dependiente del Ministerio de Cultura de España, ofrece una cifra de 175 resultados combinando los términos historia y enseñanza (se incluye historia del arte, y de la literatura), en edición electrónica, entre los que el grupo mayor lo constituyen materiales de apoyo a libros de texto de educación primaria y secundaria. Otros ejemplos en libros de texto alemanes: GuTJAHR, Hans-Joachim [Hrsg.]; FESSER, Gerd Geschichte: (Buch: alle wichtigen Unterrichtsinhalte - kurz und knap; CD-ROM: ausfïbrliche Themen mit multimedialen Elementen; Internet: Aktualisierung und Erweiterung des Wissens\}. Berlin: Paetec, 2003, 464 p. + 1 CD-ROM GUTJAHR, Hans-Joachim: Basiswissen Schule Geschichte: (Buch: alle wichtigen Unterrichtsinhalte - kurz und knapp; CD-ROM: ausfïbrliche Themen mit multimedialen Elementen; Internet: Aktualisierung und Erweiterung des Wissens\}. Mannheim: Dudenverl 2003, 464 p. + CD-ROM.

38 Romero Morante, Jesús: «De los medios informaticos y los fines educativos en la enseñanza de la Historia». Iber. Didáctica de las Ciencias Sociales, Geografía e Historia, (4), 1995, pp. 121-134. Esta misma revista Iber, dedicó su núm. 31 (Enero-febrero, 2002) 126 p., a la enseñanza de Historia con nuevas tecnologías. Contiene: Equipo directivo de Íber: «Conté: Internet y la enseñanza de la historia». PRATS, Joaquim: «Internet en las aulas de educación secundaria», pp. 716. Hernández SÁnCHEZ, Fernando; DonCel LóPeZ, Almudena: «Internet y didáctica de la historia», pp. 18-23. ItURBE POlO, Guillermo; LóPez BATAlla, Ramón; LORENZO MAGallón, Isabel: «Internet: luces y sombras en el aula», pp. 24-32. SOLER, Joaquín: «Nuevos medios: investigar y publicar historias hoy», pp. 33-49. LÓPEZ SERRA, Alfredo: «Jugar, debatir, conspirar. Medios tecnológicos y fines educativos en la enseñanza de las ciencias sociales». pp. 50-62. 
gobiernos están invirtiendo en desarrollar herramientas distribuidas para apoyo a la docencia, como es el caso del proyecto alemán Prometheus, consistente en un banco de imagen digital para arqueología e historia del arte ${ }^{39}$, o los diferentes materiales accesibles en los portales españoles dependientes de las autoridades responsables de la educación básica ${ }^{40}$. Frente a las monografías que consideran positiva la experiencia lectiva en Historia con apoyo de las nuevas tecnologías ${ }^{41}$, sin embargo, se han publicado testimonios que muestran que su aplicación en la enseñanza secundaria no ha conseguido aún cambios significativos en los métodos de aprendizaje, en lo que a Historia se refiere, o que servirse sólo de Internet obtiene resultados mediocres frente a la docencia tradicional impartida por profesores con apoyo en lecturas ${ }^{42}$. La inversión exigida

CARABIAS, Ana María: «Recursos de historia moderna en Internet: útiles para enseñar y aprender», pp. 67-77. Biosca, Eloi; CANTARell, Elena; SANCHO, Marta; VinYOles, Teresa: «Reconstruyendo el pasado. Enseñar con tecnologías de realidad virtual», pp. 103-111. BlanCO ReBOLLO, Ángel: «Las ciudades medievales y los gremios. Una experiencia didáctica en secundaria y bachillerato», Aula Historia Social (11), 2002, pp. 61-74. PraTs, Joaquím; TORRE, José Luis de la: «Recursos de Internet para la enseñanza de la historia», Iber. Didáctica de las Ciencias Sociales, Geografía e Historia, (41), 2004, pp. 5-7. Albert, J. Miquel: «Internet y la didáctica de ciencias sociales», Aula de Innovación Educativa, (139), 2005, pp. 72-75.

39 Hohmann, G; Simon, H., Verstegen, U.: «Prometheus - The distributed digital image archive for research and academic teaching and learning» NFD Information-Wissenschaft und Praxis 53 (6), ag-sept. 2002, pp. 355-360.

40 Con el Plan de Nuevas Tecnologías en la Educación, del Ministerio de Educación y Ciencia a la cabeza. Véase su portal en http://www.pntic.mec.es, sede del Centro Nacional de Información y Comunicación Educativa. Clasificados primero por etapas formativas, encontramos diversos enlaces que ofrecen materiales de apoyo a las ciencias sociales, incluyendo en ellas a la Historia, algunos de los cuales han sido merecedores de premios por el MEC. Las comunidades autónomas también ofrecen este tipo de materiales, elaborados por lo general por profesores de estos niveles. Véase el de la Generalidad de Cataluña en http://www.xtec.cat/recursos/socials/index.htm con diversos apartados relativos a Historia universal, de Cataluña o del Arte, con enlaces a diversas instituciones tanto españolas como extranjeras. Entre las iniciativas de apoyo a la docencia de Historia con las nuevas tecnologías destaca el proyecto Clío, http://clio.rediris.es que funciona desde 1998 en la forma de una revista cuyos artículos suponen una oferta de materiales que podrían servir como apoyo a los docentes de Historia en los diferentes niveles académicos. Para búsqueda de recursos educativos es muy recomendable una visita a la página del Centro de Investigación y Documentación Educativa, CIDE, del Ministerio de Educación y Ciencia, en http://www.mec.es/cide/. Merece un comentario positivo el proyecto E-HELP, European History E-Learning Project, iniciado en 2004, para mejorar el uso de las TIC e Internet en la enseñanza de historia en secundaria. Su contenido y participantes se describen en http://www.historiasiglo20.org/EHELP/index.htm.

41 TRINKLE, Dennis A.: Writing, teaching, and researching history in the electronic age: historians and computers, Armonk, N.Y.: M.E. Sharpe, 1998, XI+ 267 pp. TrinkLE, Dennis A.; Merriman, Scott A. (eds.) History.edu: essays on teaching with technology Armonk, N.Y.: M.E. Sharpe, 2000: XVIII+266 pp. (reed. en 2001). CANTU, D. Antonio and WARREN, Wilson J.: Teaching History in the Digital Classroom, Armonk, N.Y.: M. E. Sharpe, 2003. 361 pp.

42 FauX, T.L.; Black-Hughes, C.: "A comparison of using the Internet versus lectures to teach social work history» Research on Social Work Practice, 10 (4) 2000, pp. 454-466. W ALSH, Ben: 
para dotar de ordenadores las aulas, así como para mantenerlas y actualizarlas, aparte de lo necesario para adquirir materiales multimedia, ha limitado las supuestas ventajas de las tecnologías de la información. Por otra parte, hasta que el manejo de los nuevos medios ha conseguido ser fácil de aprender y sus herramientas resultan productivas ${ }^{43}$, se ha comprobado que los profesores de ciencias y de asignaturas relativas a tecnología encontraban más natural su acercamiento a los ordenadores y a todo lo que llevan consigo, que los docentes en humanidades. Si nos centramos en Historia, en niveles básicos, en los que los alumnos no han desarrollado aún un espíritu crítico, sumergirse en el océano de datos que se ofrecen desde Internet puede ser incluso perjudicial y un freno para el desarrollo en los programas lectivos convencionales, pues se requiere mucho más tiempo para valorar y clarificar los datos encontrados en la red, que para asimilar un contenido ya preparado en el marco de un temario ajustado al curso y a la edad ${ }^{44}$. Las ediciones en soporte multimedia con información histórica y objetivos docentes han proliferado desde la segunda mitad de los años 90 , en muchos países ${ }^{45}$, pero no han desbancado al libro de texto en modo alguno. Aprovechando la popularidad de juegos de simulación, como Ages of Empires o Civilizations, se podría transmitir una visión global y dinámica sobre la evolución histórica, que es imposible de encontrar de igual forma en los libros, pero la eficacia lectiva de estos programas, especialmente con alumnos de poca edad, resulta problemática ${ }^{46}$.

«A Complex Empire: National Archives learning curve takes on the British Empire» Teaching 2003, (112) pp. 22-27. Hillis, P.; MunRo, B.: «ICT in history education - Scotland and Europe» Social Science Computer Review, 23(2), Sum 2005, pp 190-205.

43 Por ejemplo, mediante programas de presentaciones gráficas, del estilo de Power Point, o editores de texto potentes capaces de integrar texto, imágenes y gráficos.

44 VAN HARTESVELDT, Fred R.: «The undergraduate research paper and electronic resources: a cautionary tale», Teaching History: A Journal of Methods 23(2), 1998, pp. 51-59. Este autor reflejaba que las facilidades de Internet para aportar grandes cantidades de datos resultaban mucho menos eficaces en el aprendizaje que el uso de métodos docentes tradicionales. La misma impresión la expresa FITCH, Nancy: «History after the web: teaching with hypermedia», History Teacher 30(4), 1997, pp. 427-441.

45 Como muestra, un par de ejemplos, uno norteamericano y otro ruso. Evans, CHARLes T. and BROWN, Robert: «Teaching the history survey course using multimedia techniques», Perspectives: American Historical Association Newsletter 36(2), 1998, pp. 17-20. ANTONOVA, Tat'iana Sergeevna and Bataeva, Tamara Vasil'evna: «Pervyi komp'iuternyi uchebnik 'Istoriia Rossii. XX vek'» [The first electronic textbook: The History of Russia in the 20th Century], Prepodavanie Istorii $v$ Shkole [Russia] (4)1998, pp. 57-67. Para España, en la base de datos DocE (http://www.eurosur.org/DOCE/ informa.html), se recogían 83 ediciones en CD-ROM interesantes para la enseñanza de historia, publicados la mayoría durante la década de 1990.

46 OsWALT, Vadim: «Elektronische Medien für den Geschichtsunterricht - Eine Herausforderung für die Geschichtsdidaktik?» International Society for History Didactics. Information 21(1), 2000, pp. 62-68. TAYLOR, Tom: «Historical simulations and the future of the historical narrative», Journal of the Association for History and Computing, 6(2), 2003. Entre las escasas experiencias sobre los juegos como material lectivo de apoyo en Historia, véase Cuenca López, José María: “Iter-itineris’: un 
En los niveles universitarios se pueden documentar experiencias innovadoras, como la desarrollada en la Georgia University donde se facilitó el uso de ordenadores portátiles y conexiones para relacionar en mayor medida a los alumnos con sus compañeros y con el profesor en un programa de Historia. Aparte de los problemas tecnológicos que presentaba, el proyecto ofreció resultados contradictorios, pues mientras no mejoraba el nivel de aprobados o la habilidad en la redacción, facilitó que algunos alumnos se introdujeran con más intensidad en el estudio de la asignatura ${ }^{47}$. En la literatura especializada no es difícil encontrar la mención a proyectos educativos que se han puesto en marcha en diversos países, como el denominado "Teaching and Learning in the Digital Age: Reconceptualizing the Introductory Survey Course”, implantado en una docena de facultades de Historia de California y Carolina del Norte ${ }^{48}$. El seguimiento de un curso de introducción a la Historia del Mundo, impartido en la Universidad Carnegie Mellon entre 2000 y 2003, demostró la preferencia de los estudiantes por las lecturas de textos, que pueden anotar, extractar o comentar, en lugar de estudiar el temario a partir de páginas web ${ }^{49}$, aunque otras experiencias valoran positivamente el trabajo con información obtenida en Internet como complemento a un programa lectivo, para temas concretos ${ }^{50}$. En otros casos, la tecnología ha permitido establecer un foro en el que el profesor plantea temas semanales a los estudiantes para que los documenten, debatan, trabajen en grupo, comparen sus aportaciones, consiguiendo un mayor estímulo intelectual y elevando el grado de participación ${ }^{51}$. Por lo pronto, son cada vez más los profesores universitarios responsables de cursos presenciales que publican su programa en Internet, junto con bibliografías, materiales de

ejemplo de juego on-line para el aprendizaje de la historia», Iber. Didáctica de las Ciencias Sociales, Geografía e Historia, (41), 2004, pp. 68-76.

47 Musselwhite, Laura G.: «Gems from rough stones: teaching with technology in the twoyear environment», History Teacher 33(1),1999 pp. 33-39.

48 Huehner, David Robert and Kallgren, Daniel. «Technology and student-centered learning: The University of Wisconsin Student History Network Project», History Teacher 33(1), 1999, pp. 41-54. FranKel, Noralee and Shapiro: «A collaboration of two- and four-year colleges: reconceptualizing the introductory survey course», History Teacher 33(1), 1999, pp. 91-95.

49 LONGHURST, James: «World history on the world wide web: a student satisfaction survey and a blinding flash of the obvious», History Teacher 36(3), 2003, pp. 343-356.

50 SEED, Patricia: «Teaching with the web: two approaches». Perspectives: American Historical Association Newsletter 36(2), 1998, pp. 9-12. El objetivo de la actividad en Internet consistía en comparar la cobertura informativa de los medios de comunicación en acontecimientos de Estados Unidos frente a los de América Latina. Véase también el proyecto TEAM desarrollarlo en la Universidad de Barcelona, en RodríGUez IlLERA, J.L.; SUAU, J. (eds.): Tecnologias multimedia para la enseñanza y aprendizaje en la universidad: el proyecto TEAM de la Universidad de Barcelona. Barcelona: Edicions de la U.B., 2003, 166 pp. + 1 CD-ROM.

51 ZARATE, Eloy: "Cyberspace, scholarship, and survey courses: a prototype for teaching World History", History Teacher 32(1), 1998, pp. 57-65. Relata la experiencia de un curso piloto desarrollado en la University of California in Riverside. 
lectura y otros elementos de refuerzo para que sus alumnos los tengan disponibles, en la misma línea que ya se practicaba en cursos a distancia.

La apertura de archivos contemporáneos con documentos digitalizados está cambiando la forma de acercarse a la red, abriendo una nueva forma de investigar y afrontar los problemas históricos ${ }^{52}$. En el aula, disponer de acceso directo a documentación primaria digitalizada permite organizar experiencias interesantes, y así lo han entendido las autoridades británicas y norteamericanas, que disponen en los portales de acceso a sus archivos nacionales de un completo programa de apoyo a la docencia en historia en los niveles de enseñanza obligatoria, incluyendo transcripciones e imágenes de los documentos originales, que sirven como ilustración para actividades de comentario de textos y profundización $\mathrm{n}^{53}$, llegando más lejos en su pretensión didáctica que una simple exposición virtual.

Antes de dejar la palabra a los especialistas que han tenido la gentileza de aportar sus contribuciones a esta sección monográficade Hispania ${ }^{54}$, conviene resaltar algunos hechos que no son obvios en este esbozo urgente de la producción bibliográfica internacional en la que los historiadores han analizado su posición en Internet. El primero consiste en constatar la madurez alcanzada por Internet, tanto en el crecimiento de sus contenidos, como en el acceso a ellos cada vez más generalizado, pues si en los años finales del siglo XX abundaban artículos o monografías orientados a ofrecer un panorama más o menos general dirigido a neófitos sobre los sitios y páginas que iban apareciendo como novedades, al tiempo que se hacía una profesión de fe en las nuevas tecnologías de la información, al día de hoy carece de sentido continuar en esta dirección, pues el ritmo de crecimiento de información en la red resulta difícil de seguir en artículos impresos, que pronto quedan desfasados. Dar noticia de páginas o novedades en Internet es una información que se encuentra mejor en la propia red que en publicaciones tradicionales, pues en las páginas web los enlaces están activos para introducirnos inmediatamente en los destinos seleccionados. No es que haya pasado la moda militante de algunos historiadores vanguardistas comprometidos en utilizar Internet, sino que las facilidades de su uso han convertido esa postura en innecesaria. Tan sólo es preciso seguir haciendo lo que el historiador está acostumbrado a hacer: plantear su discurso historiográfico y difundirlo por los canales más eficaces, normalmente las publicaciones científi-

52 Como ocurre cuando se han desclasificado y puesto en la red documentos sobre la Guerra Fría en archivos norteamericanos, europeos o chinos. GRIFFITH, Robert «Un-tangling the web of cold war studies; or, how one historian stopped worrying and learned to love the internet», Journal for Multimedia History, 3, 2000.

53 Digital Classroom, en los archivos federales norteamericanos, NARA, http://www.archives.gov/ y en National Archives del Reino Unido, especialmente su programa Learning Curve, en http://www. nationalarchives.gov.uk/default.htm.

54 La idea de reflexionar sobre estas cuestiones surgió inicialmente a partir de un seminario puesto en Internet a finales de 2001, realizado en colaboración con la Fundación Carlos de Amberes. Sus materiales se publicaron en http://www.moderna.ih.csic.es/fcamberes/. 
cas, que hoy circulan perfectamente por Internet, al objeto de que otros las lean, comenten, debatan y citen.

En segundo lugar, la calidad intrínseca de los contenidos ha de ser el principal valor que debe perseguirse, y al que se abonan todos los profesionales rigurosos, aunque no por el hecho de que algo esté en la red lleva implícito un sello de calidad, más bien suele suceder lo contrario. Por ello, además de que hayan aparecido nuevas publicaciones que sólo se editan en formato electrónico y en Internet, cada día más revistas científicas tienden a convertirse también en digitales, sin abandonar los criterios de calidad exigibles, tanto en las formas como en el contenido. De quién depende un servidor en Internet y cómo se publica en él son elementos fundamentales para establecer la credibilidad de la información que encontremos en la red. En tercer lugar, el nuevo medio va conociéndose cada vez mejor, hay historiadores jóvenes a los que no les supone apenas esfuerzo escribir en la red, pero el discurso no debería ser el mismo, intentando ir más allá que situar versiones electrónicas que reproduzcan publicaciones en papel en una dirección en Internet. Se está aprendiendo la lección de que la gestión de bases de datos, las exposiciones virtuales y los materiales docentes bien construidos requieren una infraestructura más allá de la informática, con el concurso de profesionales de diversas áreas (infografía, comunicación, diseño), que además manejen los conceptos nuevos como usabilidad, hipertexto, navegación y otros que han de aplicarse en conjunción con la información proporcionada por el historiador. La distancia que hay entre un texto literario, un guión cinematográfico que se inspire en él, y finalmente una película, puede ser similar a la que se encuentra entre una monografía histórica y una producción multimedia desarrollada sobre su contenido, con destino a Internet. El discurso creado para el nuevo medio debería despertar interés y transmitir conocimiento, más allá de lo que puede hacer el libro impreso. Por eso en algunas universidades se ofrecen programas que incluyen el aprendizaje en profundidad de los nuevos medios como apoyo a una formación tradicional en ciencias históricas o humanidades ${ }^{55}$. La posibilidad de recibir comentarios y analizar cómo se visitan las diversas páginas que componen un portal en Internet permite mejorar el diseño, corregir los errores, en fin, adaptarse a la audiencia. Los historiadores, no obstante algunas meritorias incursiones en la línea apuntada, prefieren aún hoy en día mayoritariamente difundir sus investigaciones mediante los canales académicos habituales: monografías, obras colectivas, revistas y actas de congresos. Por último, y este valor es quizá el más importante, la implantación de la red a escala planetaria puede poner al alcance de quien sea capaz de leer o entender los mensajes el contenido del discurso, sin importar las distancias. De ahí que se requiera utilizar idiomas aceptados en la comunicación académica (con preferencia el inglés), para conseguir mayor

55 Véase el Digital Humanities MA, del King's College de Londres, en http://www.kcl.ac.uk/ pgp06/programme/100, o los cursos del Maryland Institute for Technologies in the Humanities, en http://www.mith2.umd.edu. 
difusión, y para establecer redes con historiadores situados más allá de los límites nacionales y lingüísticos.

La red global de información, pese a su juventud, manifiesta una potencia imparable, se la considera ya entre los servicios básicos que ha de tener una sociedad moderna y desarrollada. Los especialistas que han colaborado en este informe han mantenido un gran interés por el nuevo medio desde su aparición. Cruz Rubio Liniers, como responsable del área de Historia en el Centro de Información y Documentación del CINDOC (CSIC), aparte haber impartido numerosos cursos en sistemas de información aplicados a la investigación y docencia en ciencias históricas, ha redactado un informe con los recursos imprescindibles en el campo de las referencias bibliográficas para los historiadores. Antonio Malalana Ureña, medievalista de formación, pero experimentado profesor en nuevas tecnologías y documentación en la Universidad San PabloCEU, ha recorrido de forma crítica el área de Historia Medieval en Internet, para ofrecer una visión de luces y sombras sobre lo que los historiadores pueden encontrar. Cómo acercarse a los archivos, especialmente desde una perspectiva didáctica ha sido el objetivo de Alberto Angulo, profesor de Historia Moderna en la Universidad del País Vasco, preocupado porque sus alumnos valoren las posibilidades que se abren en los nuevos canales de información. Finalmente, Anaclet Pons, profesor de Historia Contemporánea en la Universidad de Valencia, responsable de uno de los portales dedicados a Historia con mayor éxito y número de enlaces desde hace muchos años, aporta una reflexión ciertamente útil para el profesional que se acerca al estudio del pasado con el bagaje propio de un científico social en la era de Internet ${ }^{56}$.

56 El título de este monográfico es una adaptación intencionada y tributaria de Dennis A. Trinkle (véase nota 41 ). 